\title{
Self-consistent Full-band Modeling of Quantum Semiconductor Nanostructures
}

\author{
FRANCESCO CHIRICO, ALDO DI CARLO and PAOLO LUGLI* \\ INFM-Dipartimento di Ingegneria Elettronica, Università di Roma "Tor Vergata", 00133 Roma, Italy

\begin{abstract}
We have developed a full-band pseudopotential-based approach to describe semiconductor nanostructures. The method relies on the bulk Bloch functions expansion of the system wavefunction, which guarantee an efficient integration of the full-band approach in self-consistent schemes where Schroedinger and Poisson equations are solved iteratively. In order to gain efficiency of the method a suitable separation between structure dependent and material dependent contributions to the system hamiltonian is presented. Results are shown for a typical $\mathrm{Si} / \mathrm{SiO}_{2} \mathrm{MOS}$ structure.
\end{abstract}

Keywords: Nanostructures; HEMT; Full-band; Self-consistency; Empirical pseudopotential

\section{INTRODUCTION}

Several semiconductor devices exploit the confinement of electrons in spatial regions of the order of few nanometers. This is the case, for example, of Silicon MOS or III-V HEMTs. Such confinement, and related size quantization, are achieved using semiconductor heterojunctions, an ingredient also common to optoelectronic devices, such as lasers and modulators.

Typically, carrier confinement in semiconductor nanostructures is described in terms of $\mathbf{k} \cdot \mathbf{p}$ expansion within the Envelope Function Approximation (EFA). This "standard model", however, is not capable of describing the system in the entire Brillouin zone and loses validity for ultrasmall systems.
All the limitations of the EFA can be overcome by using full-band methods. In our approach we describe the semiconductor nanostructure in term of empirical pseudopotentials [1]. Although only few parameters are needed a large number of plane waves is required to accurately describe the system even in the bulk case. Thus, no guarantee exist that such approach can be easily extended to nanostructures where dimension of hundred of $\AA$ are typical. The purpose of this article is to present a method that maintains both the degree of physical insight of full band approaches and the speed of typical effective mass models. The method, based on the bulk Bloch functions expansion of the system wavefunction [2-8] allows us to efficiently incorporate the full-band

*Corresponding author. 
approach into self-consistent schemes where Schroedinger and Poisson equations are solved iteratively.

\section{THEORY}

The one-electron Hamiltonian for a generic nanostructure in the presence of an external potential $V(\mathbf{r})$, is given by

$\mathcal{H}=-\frac{\hbar^{2}}{2 m} \nabla^{2}+\sum_{\sigma, \alpha} W_{\alpha}^{\sigma}(\mathbf{r}) V_{\alpha}^{\sigma}\left(\mathbf{r}-\mathbf{d}_{\alpha}\right)+V(\mathbf{r})$

where $\sigma$ is the material index, $\alpha$ is the atomic base index, $\mathbf{d}_{\alpha}$, the $\alpha$-th atom offset in the unit cell, $W_{\alpha}^{\sigma}(\mathbf{r})$ a weight function [7] that selects the atom type and $V_{\alpha}^{\sigma}(\mathbf{r})$ the (periodic) local atomic pseudopotential in real space related to the $\alpha$-th atom of material $\sigma$. We are assuming that all materials constituting the structure have the same Bravais lattice although the approach can be generalized to strained materials $[9,6]$.

We expand the system wavefunction $\psi(\mathbf{r})$ over a linear combination of the bulk Bloch wavefunctions $\phi_{n \mathbf{k}}^{\sigma}(\mathbf{r})$ of all the materials forming the nanostructure

$$
\psi(\mathbf{r})=\frac{1}{\sqrt{N}} \sum_{n \mathbf{k} \sigma} C_{n \mathbf{k}}^{\sigma} \phi_{n \mathbf{k}}^{\sigma}(\mathbf{r})
$$

where $N$ is the number of unit cells in the large supercell which contains our nanostructure, $n$ the band index and $\mathbf{k}$ the wave vector. In a plane wave expansion, the bulk Bloch wavefunction are written as

$$
\phi_{n \mathbf{k}}^{\sigma}(\mathbf{r})=\langle\mathbf{r} \mid n \mathbf{k} \sigma\rangle=\frac{1}{\sqrt{\Omega_{0}}} \sum_{\mathbf{G}} B_{n \mathbf{k}}^{\sigma}(\mathbf{G}) e^{i(\mathbf{G}+\mathbf{k}) \cdot \mathbf{r}}
$$

with $\mathbf{G}$ the reciprocal lattice vector and $\Omega_{0}$ the volume of the unit cell.

By using Eqs. (2) and (3) and assuming a proper representation of the external potential the Schrödinger equation for the nanostructure can be written as a generalized eigenvalues problem [6]:

$$
\sum_{n \mathbf{k} \sigma} \mathcal{H}_{n^{\prime} \mathbf{k}^{\prime} \sigma^{\prime}, n \mathbf{k} \sigma} C_{n \mathbf{k}}^{\sigma}=E \sum_{n \mathbf{k} \sigma} \mathcal{S}_{n^{\prime} \mathbf{k}^{\prime} \sigma^{\prime}, n \mathbf{k} \sigma} C_{n \mathbf{k}}^{\sigma}
$$

where

$$
\mathcal{S}_{n^{\prime} \mathbf{k}^{\prime} \sigma^{\prime}, n \mathbf{k} \sigma}=\left\langle n^{\prime} \mathbf{k}^{\prime} \sigma^{\prime} \mid n \mathbf{k} \sigma\right\rangle=\delta_{\mathbf{k} \mathbf{k}^{\prime}} \sum_{\mathbf{G}} B_{n^{\prime} \mathbf{k}}^{\sigma^{\prime}}(\mathbf{G})^{*} B_{n \mathbf{k}}^{\sigma}(\mathbf{G})
$$

represents the bulk wavefunction overlap (equal to $\delta_{n^{\prime} \mathbf{k}^{\prime}, n \mathbf{k}}$ only if $\sigma^{\prime}=\sigma$ ).

The hamiltonian matrix element is given by:

$$
\begin{aligned}
\left\langle n^{\prime} \mathbf{k}^{\prime} \sigma^{\prime}|\mathcal{H}| n \mathbf{k} \sigma\right\rangle \\
=\mathcal{A}_{n^{\prime} \mathbf{k}^{\prime} \sigma^{\prime}, n \mathbf{k} \sigma}+ \\
\quad+\sum_{\alpha, \sigma^{\prime \prime}} W_{\alpha}^{\sigma^{\prime \prime}}\left(\mathbf{k}-\mathbf{k}^{\prime}\right) \mathcal{B}_{n^{\prime} \mathbf{k}^{\prime} \sigma^{\prime}, n \mathbf{k} \sigma}^{\alpha}+ \\
\quad+V\left(\mathbf{k}-\mathbf{k}^{\prime}\right) \mathcal{C}_{n^{\prime} \mathbf{k}^{\prime} \sigma^{\prime}, n \mathbf{k} \sigma}
\end{aligned}
$$

where $W_{\alpha}^{\sigma^{\prime \prime}}(\mathbf{k})$ and $V(\mathbf{k})$ are the discrete Fourier transform (DFT) of the $W$ and $V$ terms in Eq. (1), while the expression for the $\mathcal{A}, \mathcal{B}$ and $\mathcal{C}$ are obtained by direct evaluation [10]. The $\mathcal{A}, \mathcal{B}, \mathcal{C}$ and $\mathcal{S}$ matrices depend on the materials composition of the nanostructure and do not depend on the particular geometry, sequence of layers, and potential profile of the nanostructure itself. Thus, given a material set, $\mathcal{A}, \mathcal{B}, \mathcal{C}$ and $\mathcal{S}$ matrices can be precalculated and all variations in geometry and/or in the external potential (as in typical self-consistent cycle) will require only the DET of $W$ and $V$. This as an essential separation since the time spent to calculate $\mathcal{A}, \mathcal{B}, \mathcal{C}$ and $\mathcal{S}$ may be large while that to obtain $W_{\alpha}^{\sigma^{\prime \prime}}(\mathbf{k})$ and $V(\mathbf{k})$ is negligible.

The influence of space-charge effects induced by electronic free-charge rearrangement can be included in the full-band approach at a Hartree level by solving self-consistently Poisson equation [11]. In obtaining the space dependent charge density, it is necessary to consider explicitely the symmetry of the system. It always possible to define a unit cell of the (bulk) material forming the nanostructure in such a way that some basis vector belongs to the 
"perpendicular" space where the translational symmetry of the system is broken and the rest of the basis vectors belong to the "parallel" space, where the full periodicity of the crystal is preserved. A given Bravais vector can thus be decomposed in the perpendicular and parallel components $\mathbf{R}=\mathbf{R}_{\perp}+\mathbf{R}_{\|}$. For $\mathbf{R}_{\|}$a reciprocal parallel space can be defined and consequently the Brillouin zone $B Z_{\|}$for the $\mathbf{k}_{\|}$vectors $\left(\mathbf{k}=\mathbf{k}_{\perp}+\mathbf{k}_{\|}\right)$. In the case of a dots, for example, the parallel space will be empty thus the perpendicular space will coincide with the Bravais lattice $\left(\mathbf{R}=\mathbf{R}_{\perp}\right)$.

In order to reduce the microscopic oscillation between atomic planes, a macroscopic average similar to that used in the band-offset calculation [12] is considered. The averaged wave-function is thus given by

$$
\begin{aligned}
& \left|\psi\left(\mathbf{R}_{\perp}, \mathbf{k}_{\|}\right)\right|^{2} \\
& =\frac{1}{N} \sum_{\sigma^{\prime} n^{\prime} \mathbf{k}^{\prime}} \sum_{\sigma n \mathbf{k}} C_{n^{\prime} \mathbf{k}^{\prime}}^{\sigma^{\prime} *} C_{n \mathbf{k}}^{\sigma} e^{i\left(\mathbf{k}-\mathbf{k}^{\prime}\right)_{\perp} \cdot \mathbf{R}_{\perp} \times} \\
& \times\left[\sum_{\mathbf{G}} \sum_{\mathbf{G}^{\prime}} B_{n^{\prime} \mathbf{k}^{\prime}}^{\sigma^{\prime}}\left(\mathbf{G}^{\prime}\right)^{*} B_{n \mathbf{k}}^{\sigma}(\mathbf{G}) \delta_{\mathbf{G}_{\|}, \mathbf{G}_{\|}^{\prime}}\right. \\
& \left.\operatorname{sinc}\left(\mathbf{G}_{\perp}+\mathbf{k}_{\perp}-\mathbf{G}_{\perp}^{\prime}-\mathbf{k}_{\perp}^{\prime}\right)\right]
\end{aligned}
$$

The charge density $\rho\left(\mathbf{R}_{\perp}\right)$ is defined as:

$$
\begin{aligned}
\rho\left(\mathbf{R}_{\perp}\right)=-\frac{e}{(2 \pi)^{D}} \int_{B Z_{\|}} d \mathbf{k}_{\|} & \\
& \sum_{\nu}\left|\psi\left(\mathbf{R}_{\perp}, \mathbf{k}_{\|}, E_{\nu}\right)\right|^{2} \tilde{f}\left(E_{\nu}\right)
\end{aligned}
$$

where $e$ is the electron charge, $\nu$ labels the energy levels $\left(E_{\nu}\right)$ for a given $\mathrm{k}_{\|}$and $D$ the dimensionality of the parallel space. The function $\tilde{f}\left(E_{n}, E_{F}\right)$ is defined as follows

$\tilde{f}\left(E_{\nu}\right)= \begin{cases}f\left(E_{\nu}, E_{F}^{c}\right) & \text { for the conduction states } \\ 1-f\left(E_{\nu}, E_{F}^{v}\right) & \text { for the valence states }\end{cases}$ where $f\left(E_{\nu}, E_{F}\right)$ is the Fermi distribution function with a given Fermi level $E_{F}$. Electron and holes Fermi levels, $E_{F}{ }^{c}$ and $E_{F}{ }^{\nu}$ respectively, are different if the system is out of equilibrium as in presence of optical or electrical injection. $\tilde{f}\left(E_{\nu}, E_{F}\right)$ is a well behaved function which is different from zero only in the proximity of the valence and conduction band edges. Poisson and Schrödinger (Eq. 4) equations, are iteratively solved until convergence is reached. To speed-up the convergence we use a first order expansion of the method presented in Ref. [13].

\section{EXAMPLE OF FULL-BAND SELF-CONSISTENT CALCULATION}

In the following we present the results of our selfconsistent full-band approach for a $\mathrm{Si} / \mathrm{SiO}_{2}$ MOS structure. We consider MOS system grown in the [001] direction and we choose for the unit cell the following basis

$$
\begin{aligned}
& \mathbf{R}_{1}=a[0.5,0.5,0], \\
& \mathbf{R}_{2}=a[-0.5,0.5,0], \\
& \mathbf{R}_{3}=a[0,0,1] .
\end{aligned}
$$

The translational symmetry is thus broken only in the $\mathbf{R}_{3}$ direction thus allowing an easy separation between the perpendicular $\left(\mathbf{R}_{\perp}=n \mathbf{R}_{3}\right)$ and parallel $\left(\mathbf{R}_{\|}=m \mathbf{R}_{1}+l \mathbf{R}_{2}\right)$ spaces. The two-dimensional Brillouin zone has an irreducible-wedge (IW) defined by

$$
0 \leq k_{x}<\frac{2 \pi}{a} ; \quad 0 \leq k_{y}<\min \left(k_{x}, \frac{2 \pi}{a}-k_{x}\right)
$$

The $\mathbf{k}_{\|}$integration needed to calculate the charge density Eq. (8), is performed by using a special k-points sampling [14] of the irreducible wedge. However, only a small portion of the irreducible wedge will contribute to the density, namely those conduction band (or valence band when considered) states close to the Fermi level. We have found that convergent results can be 
obtained with a small number of special k-points in such regions, namely by using $3 \mathbf{k}_{\|}$points with $\left|\mathbf{k}_{\|}\right|<0.06$ and $18 \mathbf{k}_{\|}<$points with $\mid \mathbf{k}_{\|}-$ $[0.85,0] \mid<0.2$ (here we use $2 \pi / a$ units).

The full-band approach is not applied to the whole MOS. In fact the dimension of a typical MOS will be of the order of several micrometers and the potential variation will occur over this whole scale. This means that boundary condition should be imposed at the real edges of the devices. However, electrons will accumulate mainly in the quantized channel at the interface with the oxide. In order to approach the problem we have use an embedded calculation: the whole structure is divided in a quantum region where the full band approach is consider and in a classical region where quantization effects are not present. In this latter region, a classical three-dimensional
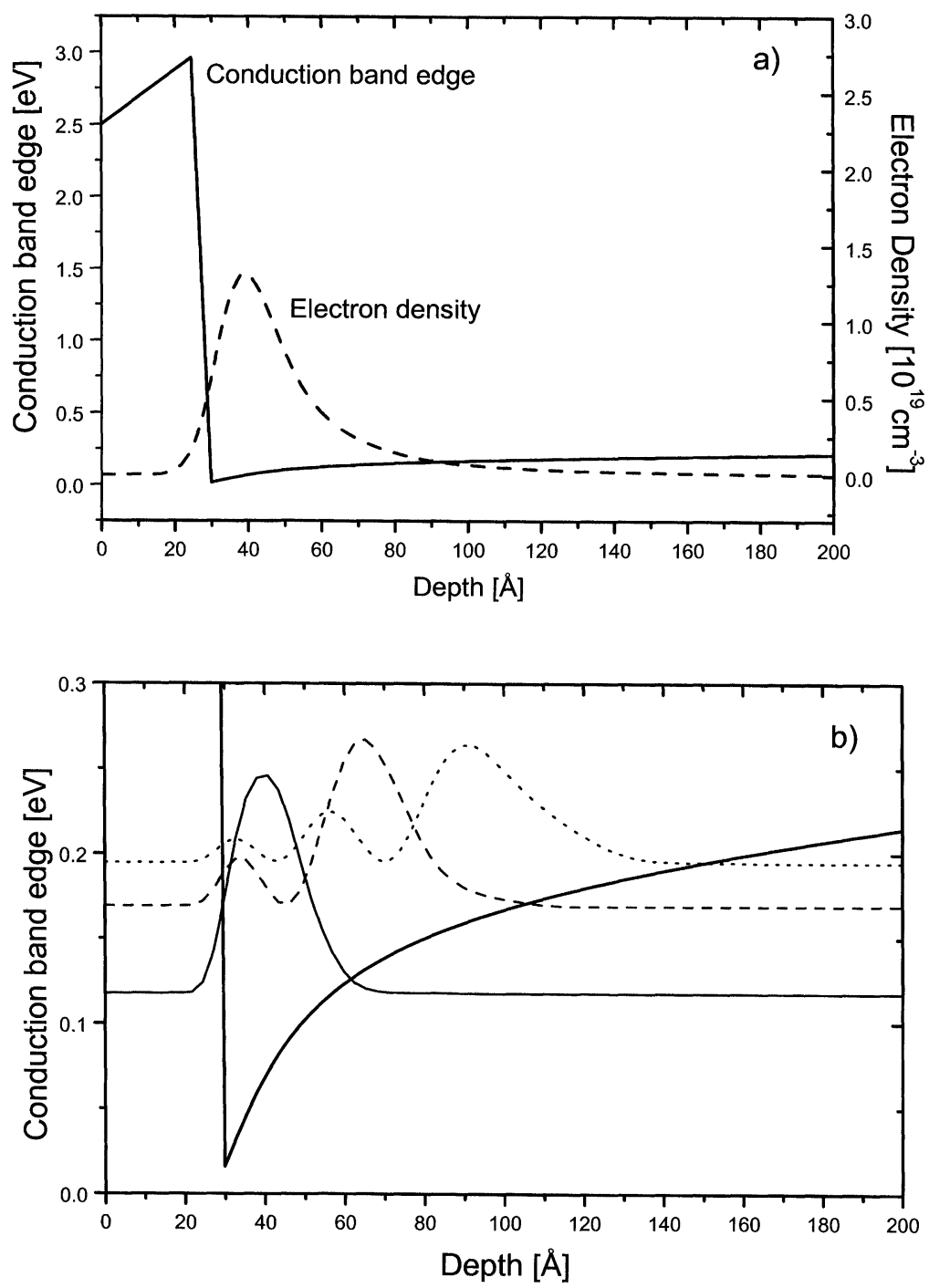

FIGURE 1 (a) Self-consistent conduction band edge profile and the electron density for the $\mathrm{Si} / \mathrm{SiO}_{2} \mathrm{MOS}$ structure. (b) Quantized channel with the first three (squared) envelope wavefunction. The zero of the envelope correspond to the energy of the quantized level. 
Thomas-Fermi description is used to calculate the electron density (if present) and ionized doping density.

We have considered a quantum region of $200 \mathrm{ML}(56.5 \mathrm{~nm})$ starting at the metal $/ \mathrm{SiO}_{2}$ interface towards the Si layer; the total width of the device is $3 \mu \mathrm{m}$. We choose $41 \mathrm{k}$-points and 2 conduction bands in the bulk band expansion for each semiconductor. In this calculation we use a pseudo- $\mathrm{SiO}_{2}$, that is we use for the insulator the same lattice structure of silicon, while the pseudopotential are tuned in order to obtain a band structure that mimics the real band structure of $\mathrm{SiO}_{2}$.

Figure 1a shows the calculated conduction band edge and the electron density. The Quantized channel with the first three (squared) envelope wavefunction is shown in Figure 1b. The zero of each wavefunction has been set to the energy of the corresponding energy level.

We should point out that the integration in the 2D-Brillouin zone in $\mathrm{k}_{\|}=[0,0]$ and $\mathrm{k}_{\|}=[0,0.85]$ $2 \pi / a$ represents the two situations where quantization arises from electron with longitudinal mass and from electrons with transversal mass in the $\mathrm{X}$ valleys.

Finally we would like to address the question of the CPU load of such calculations. Starting from a classical initial guess of the potential profile, convergency of the results of Figure 1 is obtained in 10 self-consistent cycles. The overall calculation is achieved in less than one hour in a typical singleprocessor workstation. The calculation of the $\mathcal{A}, \mathcal{B}, \mathcal{C}$ and $\mathcal{S}$ matrix element is much more time consuming and may require many hours of computation. However, as discussed previously, such matrix elements need to be calculated only once. Moreover, the structure of such matrix element call for a straightforward parallelization where each element of the matrix can be calculated in a different processor.

\section{Acknowledgements}

This work has been partially funded by MURST, CNR progetto $5 \%$ Microelettronica, and by the Office of Naval Research

\section{References}

[1] Cohen, M. L. and Chelikowsky, J. R., Electronic Structure and Optical Properties of Semiconductors, 2nd edn. Springer Ser. Solid-State Sci., 75 (Springer, Berlin, 1989).

[2] Kohn, W., In: "Solid State Physics: Advances in Research and Applications", Seits, F. and Túrnbull, D. Eds. (Academic, New York, 1957), 5, 257.

[3] Altarelli, M., In: "Heterojunction and Semiconductor Superlattices", Allan, G., Bastard, G., Boccara, N., Lannoo, M. and Voos, M. Eds. (Spronger, Berlin, 1986), p. 12.

[4] Burt, M. G. (1999). J. Phys.: Condens. Matter, 4, 6651; ibid., 11, R53 (1992).

[5] Fischetti, M. V. and Laux, S. E. (1993). Phs. Rev. B, 48, 2244.

[6] Froyen, S. (1996). J. Phys.: Condens. Matter, 8, 11029.

[7] Wang, L.-W., Franceschetti, A. and Zunger, A. (1997). Phys. Rev. Lett., 78, 2819.

[8] Foreman, B. A. (1998). Phys. Rev. Lett., 80, 3823; ibid., 81, 425 (1998).

[9] Wang, L.-W. and Zunger, A. (1999). Phys. Rev. B, 59, 15806.

[10] Chirico, F., Di Carlo, A. and Lugli, P., submitted to Phys. Rev. B.

[11] Di Carlo, A., Pescetelli, S., Paciotti, M., Lugli, P. and Graf, M. (1996). Solid State Comm., 98, 803.

[12] Baldareschi, A., Baroni, S. and Resta, R. (1988). Phys. Rev. Lett., 61, 734.

[13] Trellakis, A., Galick, A. T., Pacelli, A. and Ravaioli, U. (1997). J. Appl. Phys., 81, 7880.

[14] Froyen, S. (1989). Phys. Rev. B, 39, 3168. 

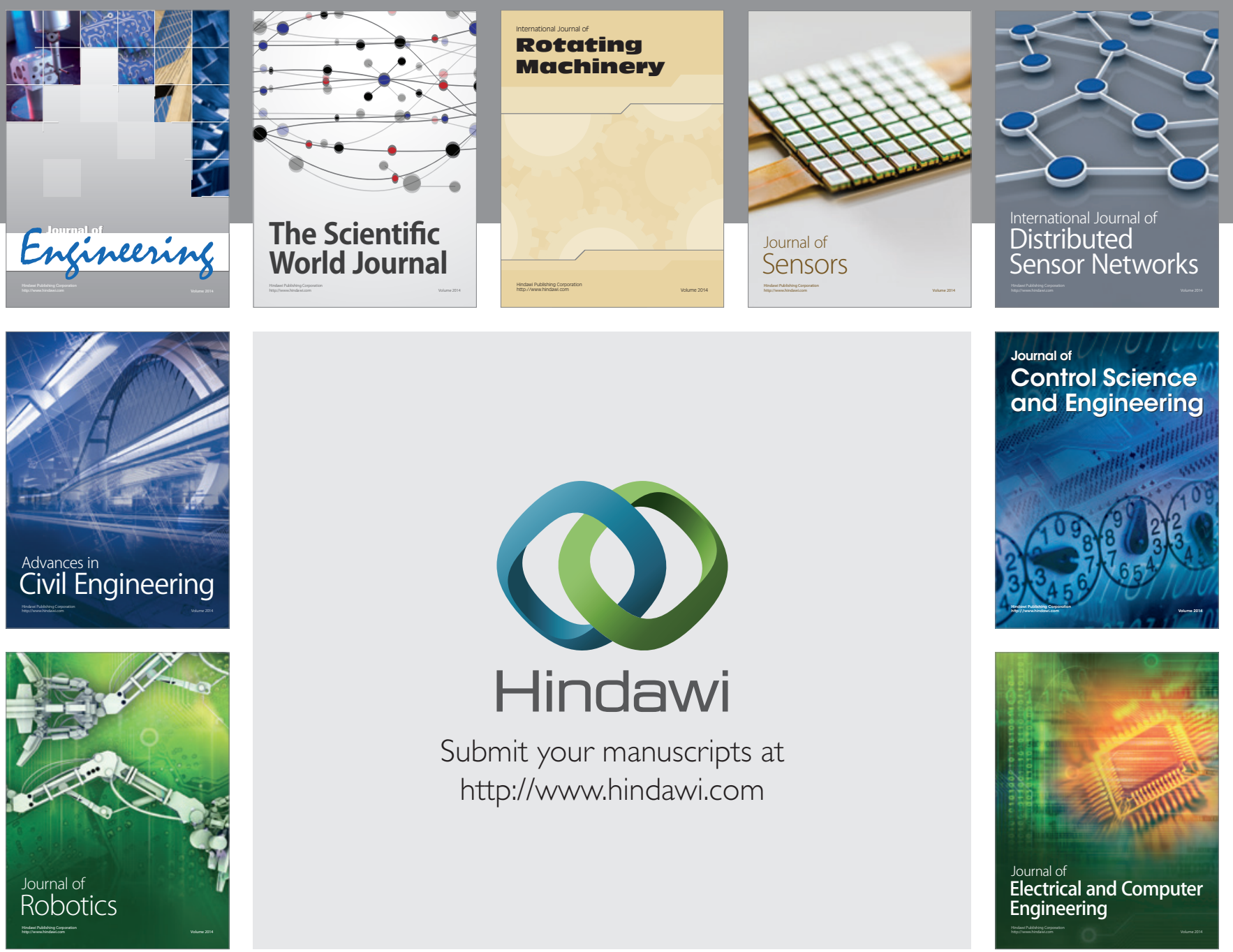

Submit your manuscripts at

http://www.hindawi.com
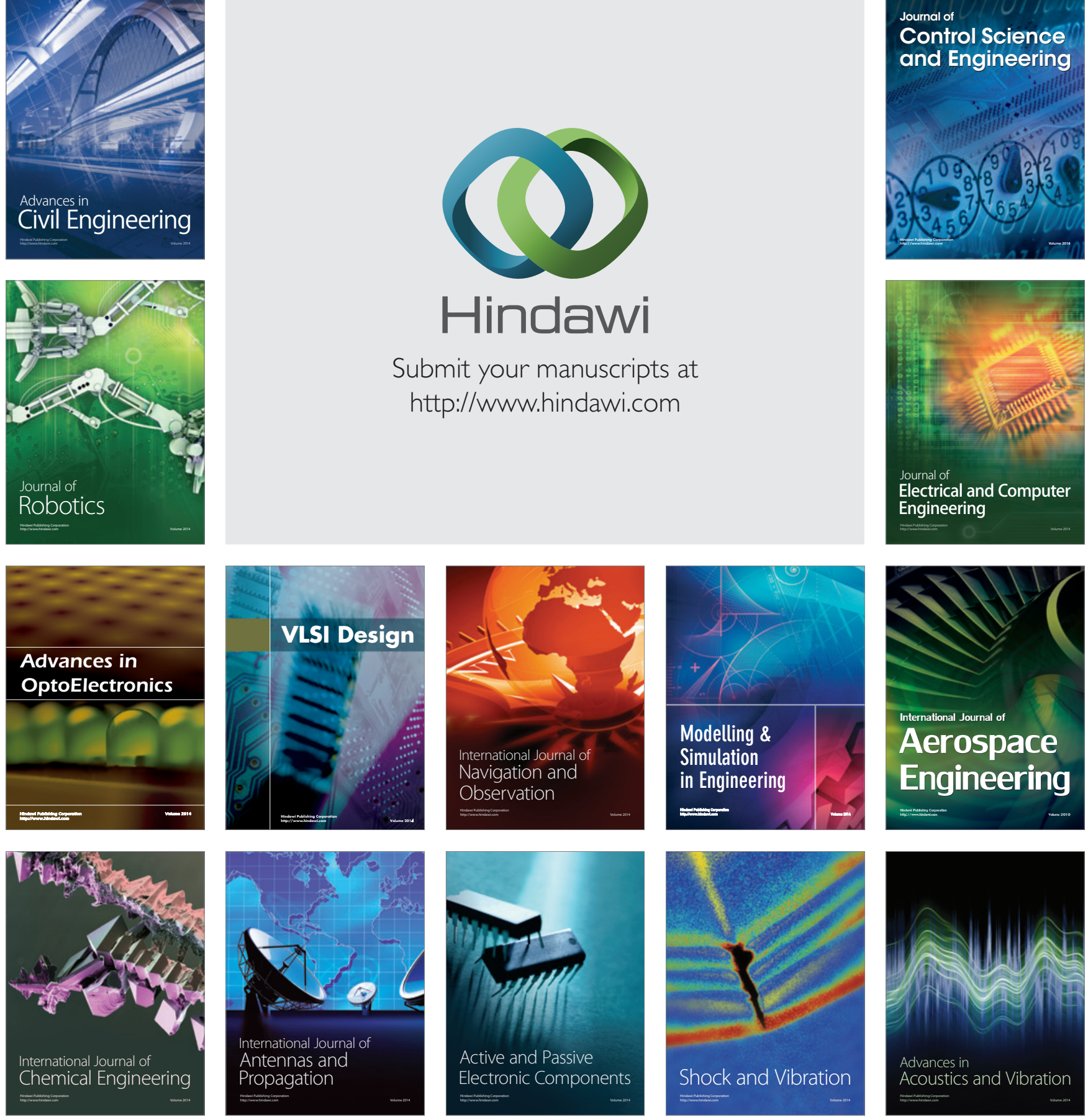